\title{
Some modern problems in structural engineering dynamics
}

\author{
I. Elishakoff* and D. Pentaras \\ Department of Mechanical Engineering, Florida Atlantic University, 777 Glades Road, Boca Raton, FL, USA
}

\begin{abstract}
This review paper deals with two problems in structural engineering dynamics; one is deterministic, the other is of stochastic nature. One problem is linear, the other is nonlinear. Authors have a biased preferential view on these problems because of their active involvement in the discussed research topics. Still, these two problems reflect, at least in a small manner, some developments in this vast and fascinating field. The first part deals with deterministic linear vibrations of double-walled carbon nanotubes either in classical or refined setting; the second part is devoted to the nonlinear random vibrations of structures.
\end{abstract}

Keywords: Deterministic linear vibrations, nonlinear random vibrations

\section{Fundamental natural frequencies of double-walled carbon nanotubes}

Nanotechnology became recently a very active field of research. This section deals with evaluation of fundamental natural frequencies of double-walled carbon nanotubes under various boundary conditions. The Bubnov-Galerkin and Petrov-Galerkin methods are applied to derive the expressions for natural frequencies. Apparently for the first time in the literature explicit expressions are obtained for the natural frequencies. These can be useful for the designer to estimate the fundamental frequency in each of two series.

\subsection{Introduction}

As Qian et al. [1] mention that "the discovery of multi-walled carbon nanotubes (MWCNTs) in 1991 has stimulated ever-broader research activities in science and engineering devoted entirely to carbon nanostructures and their applications. This is due in large part to the combination of their expected structural perfection, small size, low density, high stiffness, high strength (the tensile strength of the outer most shell of MWCNT is approximately 100 time greater that that of aluminum), and excellent electronic properties. As a result, carbon nanotubes (CNT) may find use in a wide range of application in material reinforcement, field emission pane display, chemical sensing, drug delivery, and nanoelectronics." The state of the art in modeling and simulation of nanostrucutred materials and systems was given by Gates and Hinkley [2] and Liu et al. [3] inter alia. Vibrations of double-walled carbon nanotubes (DWCNTs) have been considered in several papers. Xu et al. [4,5] and Ru [6] studied the free vibrations of a DWCNT which is composed of two coaxial single-walled CNTs interacting with each other by the interlayer van der Waals forces. Therefore, the inner and outer carbon nanotubes are modeled as two individual elastic beams [4-6]. In these studies the Euler-beam model has been used to derive exact solution for the natural frequencies at various boundary conditions. The results showed that DWCNTs have frequencies in the range of terahertz (THz). Also, in the study of vibration of carbon nanotubes, Timoshenko beam models have been used for short length-to-diameter ratios which allows for the effect of transverse shear deformation [7,8]. Likewise, the shell models have been applied recently by $\mathrm{He}$ et al. [9], Ru [10] and Wang et al. [11]. Ru [6] stresses that "carbon MWCNTs are different

\footnotetext{
*Corresponding author. E-mail: elishako@fau.edu.
} 
from traditional elastic beams due to their hollow multilayer structure and the associated interlayer van der Waals forces." The exact solutions lead to the need of solving transcendental equations. It appears that they can be usefully supplemented by simple solutions.

In this section, approximate solutions are found by using Bubnov-Galerkin [12,13] and Petrov-Galerkin [14] methods. Explicit formulas of natural frequencies are derived for the DWCNTs at different boundary conditions. Comparison of the results with recent studies shows that the above methods constitute effective alternative techniques to exact solution for studying the vibration properties of carbon nanotubes. Hereinafter we follow closely our recent paper [15] and provide some corrections.

\subsection{Analysis}

The governing differential equations for free vibration of the DWCNTs read

$$
\begin{gathered}
c_{1}\left(w_{2}-w_{1}\right)=E I_{1} \frac{\partial^{4} w_{1}}{\partial x^{4}}+\rho A_{1} \frac{\partial^{2} w_{1}}{\partial t^{2}} \\
-c_{1}\left(w_{2}-w_{1}\right)=E I_{2} \frac{\partial^{4} w_{2}}{\partial x^{4}}+\rho A_{2} \frac{\partial^{2} w_{2}}{\partial t^{2}}
\end{gathered}
$$

where $x$ is the axial coordinate, $c_{1}$ is the van der Waals interlayer interaction coefficient, $t$ is the time, $w_{j}(x, t)$ is the transverse displacement, $E$ is the Young's modulus, $I_{j}$ is the moment of inertia, $A_{j}$ is the cross-sectional area of the $j^{\text {th }}$ nanotube; the indexes $j=1,2$ denote the inner tube and outer tube, respectively.

The exact solutions for various boundary conditions were considered by $\mathrm{Xu}$ et al. [4,5]. Their derivation necessitates numerical evaluation of an $8 \times 8$ determinant and attendant cumbersome numerical analysis. Therefore, it appears imperative to obtain explicit expressions for natural frequencies by approximate methods. Here, we utilize the Bubnov-Galerkin and Petrov-Galerkin methods.

\subsection{Simply supported DWCNT: Exact solution}

For the DWCNT that is simply supported at both ends one obtains an exact solution by substitution

$$
\begin{aligned}
& w_{1}=Y_{1} \sin (m \pi \xi) \sin (\omega t) \\
& w_{2}=Y_{2} \sin (m \pi \xi) \sin (\omega t)
\end{aligned}
$$

where $\xi=x / L$ is a non-dimensional axial coordinate, and $m=1,2, \ldots$ is the number of half-waves in the longitudinal direction as well as the sequence number of the vibrational mode, $\omega$ is the sought natural frequency. We substitute Eq. (2) into Eq. (1) and demand non-triviality of $Y_{1}$ and/or $Y_{2}$. In order to have a nontrivial solution the following determinant must vanish

$$
\left|\begin{array}{cc}
E I_{1}(m \pi / L)^{4}-\rho A_{1} \omega^{2}+c_{1} & -c_{1} \\
c_{1} & -E I_{2}(m \pi / L)^{4}+\rho A_{2} \omega^{2}-c_{1}
\end{array}\right|
$$

The equation for natural frequency squared $\omega^{2}$ reads

$$
\rho^{2} A_{1} A_{2} \omega^{4}-\alpha \omega^{2}+\beta=0
$$

where

$$
\begin{aligned}
& \alpha=\rho L^{-4}\left[c_{1} L^{4}\left(A_{1}+A_{2}\right)+m^{4} \pi^{4} E\left(A_{1} I_{2}+A_{2} I_{1}\right)\right] \\
& \beta=m^{4} \pi^{4} E L^{-8}\left[c_{1} L^{4}\left(I_{1}+I_{2}\right)+m^{4} \pi^{4} E I_{1} I_{2}\right]
\end{aligned}
$$

By letting $m=1$ and solving Eq. (4), we get the following solutions for $\omega^{2}$ :

$$
\begin{aligned}
& \omega_{1, m}^{2}=\frac{\alpha-\sqrt{\alpha^{2}-4 \rho^{2} \beta A_{1} A_{2}}}{2 \rho^{2} A_{1} A_{2}} \\
& \omega_{2, m}^{2}=\frac{\alpha+\sqrt{\alpha^{2}-4 \rho^{2} \beta A_{1} A_{2}}}{2 \rho^{2} A_{1} A_{2}}
\end{aligned}
$$


Table 1

First natural frequencies in each series for various values of $L / d$ (simply supported DWCNTs at both ends)

\begin{tabular}{llllllllllll}
\hline$L / d$ & 10 & 11 & 12 & 13 & 14 & 15 & 16 & 17 & 18 & 19 \\
\hline $\begin{array}{l}\omega_{1,1} \\
\left(10^{12} \mathrm{rad} / \mathrm{sec}\right)\end{array}$ & 0.46729 & 0.38623 & 0.32456 & 0.27656 & 0.23847 & 0.20774 & 0.18259 & 0.16174 & 0.14427 & 0.12948 & 0.11686 \\
$\omega_{2,1}$ & 7.8852 & 7.8821 & 7.8802 & 7.8790 & 7.8781 & 7.8775 & 7.8771 & 7.8767 & 7.8765 & 7.8763 & 7.8762 \\
$\left(10^{12} \mathrm{rad} / \mathrm{sec}\right)$ & & & & & & & & & & & \\
\hline
\end{tabular}

The indexes 1 and 2 indicate the first and second series of frequencies, respectively. For numerical analysis hereinafter we fix the values of the Young's modulus $E$ at $1 \mathrm{TPa}$ and the mass density $\rho=2.3 \mathrm{~g} / \mathrm{cm}^{3}$ following Xu et al. [4], Yoon et al. [7], Natsuki et al. [16] and Ru [17]. Yoon et al. [7] and Ru [17] adopted the following dimensions: the mean radius of inner tube equals $0.35 \mathrm{~nm}$, the mean radius of the outer tube equals $0.7 \mathrm{~nm}$, the thickness of each nanotube equals $0.35 \mathrm{~nm}$. Noting that then $\left(R_{i}^{(1)}+R_{i}^{(2)}\right) / 2=0.35 \mathrm{~nm}, R_{i}^{(2)}-R_{i}^{(1)}=0.35 \mathrm{~nm}$, we get $R_{i}^{(1)}=$ $0.175 \mathrm{~nm}$ and $R_{i}^{(2)}=0.525 \mathrm{~nm}$. Here $R_{i}^{(1)}$ is the inner radius and $R_{i}^{(2)}$ is the outer radius of the inner tube. Likewise $\left(R_{o}^{(1)}+R_{o}^{(2)}\right) / 2=0.7 \mathrm{~nm},\left(R_{o}^{(2)}-R_{o}^{(1)}\right)=0.35 \mathrm{~nm}$, imply that $R_{o}^{(1)}=0.525 \mathrm{~nm}$ and $R_{o}^{(2)}=0.875 \mathrm{~nm}$, where $R_{o}^{(1)}$ and $R_{o}^{(2)}$ are the inner and outer radii of outer tube, respectively. However this data suggest that there is no gap between two nanotubes.

Hence we adopt Xu et al.'s data. The van der Waals interlayer interaction coefficient is fixed at $c_{1}=71.11 \mathrm{GPa}$ [4]; mean inner radius $R_{1}$ equals $0.35 \mathrm{~nm}$, whereas the mean outer radius $R_{2}$ equals $0.7 \mathrm{~nm}$; thickness $\delta_{0}$ of each tube equals $0.34 \mathrm{~nm}$. Thus, $R_{i}^{(1)}=0.18 \mathrm{~nm}, R_{i}^{(2)}=0.52 \mathrm{~nm}, R_{o}^{(1)}=0.53 \mathrm{~nm}$, and $R_{o}^{(2)}=0.87 \mathrm{~nm}$. Table 1 lists the first natural frequency in each series for various values of $L / d$, where $L$ is the beam length and $d$ is the mean diameter of the outer tube.

The value for $\omega_{1,1}=0.46729 \times 10^{12} \mathrm{rad} / \mathrm{sec}$ evaluated for $L / d=10$ is close to the value $0.46 \times 10^{12} \mathrm{rad} / \mathrm{sec}$ reported by $\mathrm{Xu}$ et al. [4]. The first natural frequency in the second series $\omega_{2,1}=7.8852 \times 10^{12} \mathrm{rad} / \mathrm{sec}$ is close to the value $\omega_{2,1}=7.71 \times 10^{12} \mathrm{rad} / \mathrm{sec}$ reported by Xu et al. [4]. Present value is 2.23 percent above Xu's result. For $L / d=20, \mathrm{Xu}$ et al. [4] report the values $\omega_{1,1}=0.11 \times 10^{12} \mathrm{rad} / \mathrm{sec}$ and $\omega_{2,1}=7.76 \times 10^{12} \mathrm{rad} / \mathrm{sec}$, respectively. These correlate well with our values of $0.12 \times 10^{12} \mathrm{rad} / \mathrm{sec}$ and $7.87 \times 10^{12} \mathrm{rad} / \mathrm{sec}$, respectively. Note that the frequencies given in $[4,5,15,16]$ should be reported in $\mathrm{rad} / \mathrm{sec}$ instead of $\mathrm{Hz}$.

The exact solution given in Eqs (4) and (5) will then serve as the benchmark solution, against which the efficacy of approximate solutions will be tested.

\subsection{Simply supported DWCNT: Bubnov-Galerkin method}

In order to ascertain the accuracy of the approximate solutions we first apply the Bubnov-Galerkin [12,13] method to the simply supported DWCNT the exact solution for which was reported in preceding section.

We approximate the displacements as follows

$$
w_{1}=D_{1} \sin (\omega t) \varphi^{(1)}, \quad w_{2}=D_{2} \sin (\omega t) \varphi^{(1)}
$$

where

$$
\varphi^{(1)}=3 \xi^{5}-10 \xi^{3}+7 \xi
$$

The function in Eq. (7) is so called Duncan polynomial [18]. We substitute the expressions (6) into governing differential Eq. (1); we then multiply the result of the substitution by $\varphi^{(1)}(\xi)$ and integrate over the length of the beam. The natural frequencies derived via approximate methods are denoted by overbar, as $\bar{\omega}$. We get the following two equations for $D_{1}$ and $D_{2}$ :

$$
\begin{array}{r}
\left(-L^{4} \rho A_{1} \bar{\omega}^{2}+L^{4} c_{1}+99 E I_{1}\right) D_{1}+-L^{4} c_{1} D_{2}=0 \\
-L^{4} c_{1} D_{1}+\left(-L^{4} \rho A_{2} \bar{\omega}^{2}+L^{4} c_{1}+99 E I_{2}\right) D_{2}=0
\end{array}
$$

We demand the determinant

$$
\left|\begin{array}{cc}
-L^{4} \rho A_{1} \bar{\omega}^{2}+L^{4} c_{1}+99 E I_{1} & -L^{4} c_{1} \\
-L^{4} c_{1} & -L^{4} \rho A_{2} \bar{\omega}^{2}+L^{4} c_{1}+99 E I_{2}
\end{array}\right|
$$


to vanish. This leads to the frequency equation

$$
\begin{aligned}
& L^{8} \rho^{2} A_{1} A_{2} \bar{\omega}^{4}+\left(-99 L^{4} A_{1} E I_{2}-L^{8} A_{1} c_{1}-99 E I_{1} L^{4} A_{2}-L^{8} c_{1} A_{2}\right) \rho \bar{\omega}^{2} \\
& \quad+99 L^{4} c_{1} E I_{2}+99 E I_{1} L^{4} c_{1}+9801 E^{2} I_{1} I_{2}=0
\end{aligned}
$$

with roots

$$
\begin{aligned}
\bar{\omega}_{1,1}^{2}= & {\left[L^{4} A_{1} c_{1}+L^{4} c_{1} A_{2}+99 A_{1} E I_{2}+99 E I_{1} A_{2}-\left(L^{8} A_{1}^{2} c_{1}^{2}+2 L^{8} A_{1} c_{1}^{2} A_{2}\right.\right.} \\
& +198 L^{4} A_{1}^{2} c_{1} E I_{2}-198 L^{4} A_{1} c_{1} E I_{1} A_{2}+L^{8} c_{1}^{2} A_{2}^{2}-198 L^{4} c_{1} A_{2} A_{1} E I_{2} \\
& +198 L^{4} c_{1} A_{2}^{2} E I_{1}+9801 A_{1}^{2} E^{2} I_{2}^{2}-19602 A_{1} E^{2} I_{2} I_{1} A_{2} \\
& \left.\left.+9801 E^{2} I_{1}^{2} A_{2}^{2}\right)^{1 / 2}\right] / 2 L^{4} \rho A_{1} A_{2} \\
\bar{\omega}_{2,1}^{2}= & {\left[L^{4} A_{1} c_{1}+L^{4} c_{1} A_{2}+99 A_{1} E I_{2}+99 E I_{1} A_{2}+\left(L^{8} A_{1}^{2} c_{1}^{2}+2 L^{8} A_{1} c_{1}^{2} A_{2}\right.\right.} \\
& +198 L^{4} A_{1}^{2} c_{1} E I_{2}-198 L^{4} A_{1} c_{1} E I_{1} A_{2}+L^{8} c_{1}^{2} A_{2}^{2}-198 L^{4} c_{1} A_{2} A_{1} E I_{2} \\
& +198 L^{4} c_{1} A_{2}^{2} E I_{1}+9801 A_{1}^{2} E^{2} I_{2}^{2}-19602 A_{1} E^{2} I_{2} I_{1} A_{2} \\
& \left.\left.+9801 E^{2} I_{1}^{2} A_{2}^{2}\right)^{1 / 2}\right] / 2 L^{4} \rho A_{1} A_{2}
\end{aligned}
$$

For the data adopted in the Section 3, for $L / d=10$ we obtain from Eq. (11) the following frequencies,

$$
\bar{\omega}_{1,1}=0.47108657 \times 10^{12} \mathrm{rad} / \mathrm{sec}, \bar{\omega}_{2,1}=7.88534526 \times 10^{12} \mathrm{rad} / \mathrm{sec}
$$

The percentagewise difference between $\bar{\omega}_{1,1}$ and the exact value in Table $1 \omega_{1,1}$ is $0.81 \%$, whereas the respective difference between $\bar{\omega}_{2,1}$ and $\omega_{2,1}$ is $0.002 \%$. This demonstrates the high accuracy of the Bubnov-Galerkin method for the DWCNTs.

Consider now another coordinate function, namely

$$
\varphi^{(2)}=\xi^{4}-2 \xi^{3}+\xi
$$

instead of $\varphi^{(1)}$ in Eq. (7). This function is due to Duncan [18] although in completely different context. The procedure as described above leads to frequency determinant

$$
\left|\begin{array}{cc}
-31 L^{4} \rho A_{1} \bar{\omega}^{2}+31 L^{4} c_{1}+3024 E I_{1} & -31 L^{4} c_{1} \\
-31 L^{4} c_{1} & -31 L^{4} \rho A_{2} \bar{\omega}^{2}+31 L^{4} c_{1}+3024 E I_{2}
\end{array}\right|
$$

and attendant frequency equation

$$
\begin{aligned}
& 961 L^{8} \rho^{2} A_{1} A_{2} \bar{\omega}^{4}-\left(93744 L^{4} A_{1} E I_{2}+961 L^{8} A_{1} c_{1}+93744 E I_{1} L^{4} A_{2}+961 L^{8} c_{1} A_{2}\right) \rho \bar{\omega}^{2} \\
& \quad+93744 L^{4} c_{1} E I_{2}+93744 E I_{1} L^{4} c_{1}+9144576 E^{2} I_{1} I_{2}=0
\end{aligned}
$$

whose roots are

$$
\begin{aligned}
\bar{\omega}_{1,1}^{2}= & {\left[31 L^{4} A_{1} c_{1}+31 L^{4} c_{1} A_{2}+3024 A_{1} E I_{2}+3024 E I_{1} A_{2}-\left(961 L^{8} A_{1}^{2} c_{1}^{2}+1922 L^{8} A_{1} c_{1}^{2} A_{2}\right.\right.} \\
& +187488 L^{4} A_{1}^{2} c_{1} E I_{2}-187488 L^{4} A_{1} c_{1} E I_{1} A_{2}^{+} 961 L^{8} c_{1}^{2} A_{2}^{2}-187488 L^{4} c_{1} A_{2} A_{1} E I_{2} \\
& +187488 L^{4} c_{1} A_{2}^{2} E I_{1}+9144576 A_{1}^{2} E^{2} I_{2}^{2}-18289152 A_{1} E^{2} I_{2} I_{1} A_{2} \\
& \left.\left.+9144576 E^{2} I_{1}^{2} A_{2}^{2}\right)^{1 / 2}\right] / 62 L^{4} \rho A_{1} A_{2} \\
\bar{\omega}_{2,1}^{2}= & {\left[31 L^{4} A_{1} c_{1}+31 L^{4} c_{1} A_{2}+3024 A_{1} E I_{2}+3024 E I_{1} A_{2}+\left(961 L^{8} A_{1}^{2} c_{1}^{2}+1922 L^{8} A_{1} c_{1}^{2} A_{2}\right.\right.} \\
& +187488 L^{4} A_{1}^{2} c_{1} E I_{2}-187488 L^{4} A_{1} c_{1} E I_{1} A_{2}^{+} 961 L^{8} c_{1}^{2} A_{2}^{2}-187488 L^{4} c_{1} A_{2} A_{1} E I_{2} \\
& +187488 L^{4} c_{1} A_{2}^{2} E I_{1}+9144576 A_{1}^{2} E^{2} I_{2}^{2}-18289152 A_{1} E^{2} I_{2} I_{1} A_{2} \\
& \left.\left.9144576 E^{2} I_{1}^{2} A_{2}^{2}\right)^{1 / 2}\right] / 62 L^{4} \rho A_{1} A_{2}
\end{aligned}
$$


The numerical values for the data listed in Section 1.2, for $L / D=10$ we obtain from Eq. (16) the following natural frequencies

$$
\bar{\omega}_{1,1}=0.46762245 \times 10^{12} \mathrm{rad} / \mathrm{sec}, \bar{\omega}_{2,1}=7.88520231 \times 10^{12} \mathrm{rad} / \mathrm{sec}
$$

It is seen that the coordinate function $\varphi^{(2}$ in Eq. (13) leads to lower value of the fundamental frequency estimate than use of the function $\varphi^{(1)}$ in Eq. (7). Therefore, the expressions in Eq. (16) are preferable to those in Eq. (11). Additionally, the percentagewise difference with the exact solution in estimation of $\bar{\omega}_{1,1}$ constitutes $0.07 \%$, whereas its counterpart for $\bar{\omega}_{2,1}$ is $0.0002 \%$. These small differences attest for the high reliability of the Bubnov-Galerkin method for studying DWCNTs.

\subsection{Simply-supported DWCNTs: Petrov-Galerkin method}

In 1940 Petrov [14] suggested a modification to the Bubnov-Galerkin method; he proposed to employ two systems of functions simultaneously; namely, one system of functions is used to approximate the displacement, whereas another set of functions is used for satisfying the orthogonality condition.

We first substitute the coordinate function $\varphi^{(2)}$ in Eq. (13) into governing equations as was done in Eq. (6). The results however are not multiplied by $\varphi^{(2)}$ as in Bubnov-Galerkin method, but by some other function. We choose this multiplicative function to be

$$
\psi=\xi^{6}-5 \xi^{3}+4 \xi
$$

Performing the Petrov-Galerkin procedure results in the following equations for $D_{1}$ and $D_{2}$ :

$$
\begin{aligned}
\left(169 L^{4} \rho A_{1} \bar{\omega}^{2}-169 L^{4} c_{1}-16500 E I_{1}\right) D_{1}+169 L^{4} c_{1} D_{2} & =0 \\
169 L^{4} c_{1} D_{1}+\left(169 L^{4} \rho A_{2} \bar{\omega}^{2}-169 L^{4} c_{1}-16500 E I_{2}\right) D_{2} & =0
\end{aligned}
$$

The frequency equation reads

$$
\begin{aligned}
& 28561 L^{8} \rho^{2} A_{1} A_{2} \bar{\omega}^{4}+\left(-2788500 L^{4} A_{1} E I_{2}-28561 L^{8} A_{1} c_{1}-2788500 E I_{1} L^{4} A_{2}-28561 L^{8} c_{1} A_{2}\right) \rho \bar{\omega}^{2} \\
& \quad+278850 L^{4} c_{1} E I_{2}+2788500 E I_{1} L^{4} c_{1}+272250000 E^{2} I_{1} I_{2}=0
\end{aligned}
$$

with roots

$$
\begin{aligned}
\bar{\omega}_{1,1}^{2}= & {\left[169 L^{4} A_{1} c_{1}+169 L^{4} c_{1} A_{2}+16500 A_{1} E I_{2}+16500 E I_{1} A_{2}-\left(28561 L^{8} A_{1}^{2} c_{1}^{2}+57122 L^{8} A_{1} c_{1}^{2} A_{2}\right.\right.} \\
& +5577000 L^{4} A_{1}^{2} c_{1} E I_{2}-5577000 L^{4} A_{1} c_{1} E I_{1} A_{2}^{+} 28561 L^{8} c_{1}^{2} A_{2}^{2}-5577000 L^{4} c_{1} A_{2} A_{1} E I_{2} \\
& +5577000 L^{4} c_{1} A_{2}^{2} E I_{1}+272250000 A_{1}^{2} E^{2} I_{2}^{2}-544500000 A_{1} E^{2} I_{2} I_{1} A_{2} \\
& \left.\left.+272250000 E^{2} I_{1}^{2} A_{2}^{2}\right)^{1 / 2}\right] / 338 L^{4} \rho A_{1} A_{2} \\
\bar{\omega}_{2,1}^{2}= & {\left[169 L^{4} A_{1} c_{1}+169 L^{4} c_{1} A_{2}+16500 A_{1} E I_{2}+16500 E I_{1} A_{2}+\left(28561 L^{8} A_{1}^{2} c_{1}^{2}+57122 L^{8} A_{1} c_{1}^{2} A_{2}\right.\right.} \\
& +5577000 L^{4} A_{1}^{2} c_{1} E I_{2}-5577000 L^{4} A_{1} c_{1} E I_{1} A_{2}^{+} 28561 L^{8} c_{1}^{2} A_{2}^{2}-5577000 L^{4} c_{1} A_{2} A_{1} E I_{2} \\
& +5577000 L^{4} c_{1} A_{2}^{2} E I_{1}+272250000 A_{1}^{2} E^{2} I_{2}^{2}-544500000 A_{1} E^{2} I_{2} I_{1} A_{2} \\
& \left.\left.+272250000 E^{2} I_{1}^{2} A_{2}^{2}\right)^{1 / 2}\right] / 338 L^{4} \rho A_{1} A_{2}
\end{aligned}
$$

Numerical evaluation of Eq. (21) yields for $L / d=10$,

$$
\bar{\omega}_{1,1}=0.46782540 \times 10^{12} \mathrm{rad} / \mathrm{sec}, \bar{\omega}_{2,1}=7.88521066 \times 10^{12} \mathrm{rad} / \mathrm{sec}
$$

Comparison of the results in Eq. (22) with the values in Eq. (17) show that $\bar{\omega}_{1,1}$ obtained by Petrov-Galerkin method is slightly higher than that given by Bubnov-Galerkin method; however, the estimates for $\bar{\omega}_{2,1}$ resulting from both the Petrov-Galerkin and Bubnov-Galerkin methods are nearly coincident.

Likewise, it is remarkable that the combinations of pair of functions

$$
\varphi^{(2)}=\xi^{4}-2 \xi^{3}+\xi, \psi=3 \xi^{5}-10 \xi^{3}+7 \xi
$$


or the one obtained by the reversal of the substitution and the multiplication functions

$$
\varphi^{(1)}=3 \xi^{5}-10 \xi^{3}+7 \xi, \quad \psi=\xi^{4}-2 \xi^{3}+\xi
$$

both yield the same results as the Bubnov-Galerkin method where the coordinate function $\varphi^{(2)}$ in Eq. (13) is used.

It must be also noted that the use of the combination

$$
\varphi^{(3)}=\sin (\pi \xi), \quad \psi=\xi^{4}-2 \xi^{3}+\xi
$$

or

$$
\varphi^{(2)}=\xi^{4}-2 \xi^{3}+\xi, \quad \psi=\sin (\pi \xi)
$$

both yield exact expressions of the natural frequency in Eq. (5). This result must have been anticipated since in Eqs (25) and (26), either the substitution function $\varphi$ or the multiplicative function $\psi$ coincides with the exact mode shape.

\section{Natural frequencies of carbon nanotubes based on simplified Bresse-Timoshenko theory}

This section deals with evaluation of the natural frequencies of double-walled carbon nanotubes based on two versions of the Bresse-Timoshenko equations that take into account shear deformation and rotary inertia. Simplified set of governing differential equations is suggested that omits the fourth order time derivative in the equations of both inner and outer nanotubes. Numerical results for simply supported carbon nanotubes suggest that the simplified set of differential equations provides excellent results.

\subsection{Introduction}

Recently, it was argued in the American press, that nanotubes may pose health risks similar to asbestos [19]. Indeed, Donaldson et al. [20] studied properties of CNT in relation to pulmonary toxicology and workplace safety. They noted that their needle-like shape is comparable to that of asbestos with possible deceases like mesothelioma or cancer of the lining or the lungs. In Ref. 17, Donaldson et al. were quoted: "Long ones are harmful, and short ones are not." These observations lead one to conclude that one ought to anticipate the more widespread use of short MWCNTs, rather than the long ones.

This poses the question on the modeling of the MWCNTs. Specifically, for the short MWCNTs, it appears that the Bresse-Timoshenko beam theory is more appropriate than the Bernoulli-Euler beam theory.

Bresse-Timoshenko beam model has been utilized recently for carbon nanotubes with short length-to-diameter ratios which allow for the effect of transverse shear deformation in several studies. Ru [6], Yoon et al. [7] and Wang et al. [21] used the Timoshenko beam model for vibration study of MWCNTs. The results from these studies showed that the frequencies of MWCNTs ranged in terahertz $(\mathrm{THz})$ scale. Comparison of these results with the results based on Euler beam theory showed a significant difference when the length-to-diameter ratios are small and the difference is expanded for higher vibration modes. Yoon et al. [22] used the Timoshenko beam theory for the study of transverse wave propagation in carbon nanotubes and Zang et al. [23] used the same theory for the buckling of MWCNTs.

\subsection{Bresse-Timoshenko equations for homogeneous beams}

In this section we briefly review the governing differential equations for the homogeneous Bresse-Timoshenko beams. Two coupled differential equations describe the vibrations of such a beam [24-26]

$$
\begin{aligned}
& -k^{\prime} A G\left(\frac{\partial \psi}{\partial x}-\frac{\partial^{2} v}{\partial x^{2}}\right)=\rho A \frac{\partial^{2} v}{\partial t^{2}} \\
& E I \frac{\partial^{2} \psi}{\partial x^{2}}-k^{\prime} A G\left(\psi-\frac{\partial v}{\partial x}\right)=\rho I \frac{\partial^{2} \psi}{\partial t^{2}}
\end{aligned}
$$


where $k^{\prime}$ is the shear coefficient which depends on the shape of the cross-section, $A$ is the area of the cross-section, $G$ is the modulus of elasticity in shear, $\psi$ is the slope of the deflection curve, $v$ is the total deflection, $x$ is the axial displacement, $\rho$ is the mass density per unit volume, $t$ is the time, $E$ is the Young's modulus of elasticity and $I$ is the moment of inertia.

Eliminating $\psi$ form Eqs (27) and (28) Weaver et al. [24-26] reduce them to a single differential equation

$$
E I \frac{\partial^{4} v}{\partial x^{4}}+\rho A \frac{\partial^{2} v}{\partial t^{2}}-\rho I\left(1+\frac{E}{k^{\prime} G}\right) \frac{\partial^{2} v}{\partial x^{2} \partial t^{2}}+\frac{\rho^{2} I}{k^{\prime} G} \frac{\partial^{4} v}{\partial t^{4}}=0
$$

For simply-supported beam the following substitution is made

$$
v=Y \sin \frac{j \pi x}{L} \sin \omega t
$$

for free vibration analysis. In Eq. (30) $j=1,2, \ldots$ is the number of half-waves in axial direction, $L=$ length, $Y=$ amplitude, $\omega=$ sought natural frequency for which the following bi-quadratic equation is obtained:

$$
E I\left(\frac{m \pi}{L}\right)^{4}-\rho A \omega^{2}-\rho I\left(1+\frac{E}{k^{\prime} G}\right)\left(\frac{m \pi}{L}\right)^{2} \omega^{2}+\frac{\rho^{2} I}{k^{\prime} G} \omega^{4}=0
$$

As is seen, Eq. (31) predicts two frequency series for each value of $m$ and other parameters fixed:

$$
\begin{aligned}
\omega_{1, m}^{2}= & \left\{A k^{\prime} G L^{2}+m^{2} \pi^{2} k^{\prime} G I+m^{2} \pi^{2} E I-\left[\left(A k^{\prime} G\right)^{2} L^{4}+2 A I\left(k^{\prime} G L m \pi\right)^{2}+2 A k^{\prime} G E I(L m \pi)^{2}\right.\right. \\
& \left.\left.+\left(I k^{\prime} G\right)^{2}(m \pi)^{4}-2 E k^{\prime} G I^{2}(m \pi)^{4}+(E I)^{2}(m \pi)^{4}\right]^{1 / 2}\right\} / 2 I \rho L^{2} \\
\omega_{2, m}^{2}= & \left\{A k^{\prime} G L^{2}+m^{2} \pi^{2} k^{\prime} G I-m^{2} \pi^{2} E I+\left[\left(A k^{\prime} G\right)^{2} L^{4}+2 A I\left(k^{\prime} G L m \pi\right)^{2}-2 A k^{\prime} G E I(L m \pi)^{2}\right.\right. \\
& \left.\left.+\left(I k^{\prime} G\right)^{2}(m \pi)^{4}-2 E k^{\prime} G I^{2}(m \pi)^{4}+(E I)^{2}(m \pi)^{4}\right]^{1 / 2}\right\} / 2 I \rho L^{2}
\end{aligned}
$$

Timoshenko $[25,26]$ shows that the last term, containing the expression $\omega^{4}$ in Eq. (31) contributes insignificantly in the evaluation of the lower series of natural frequencies $\omega_{1, m}$, in Eq. (32). Once the last term in Eq. (31) is omitted, the following expression for $\omega_{1, m}^{2}$ results

$$
\omega_{1, m}^{2}=\frac{E I m^{4} \pi^{4} G k^{\prime}}{\rho L^{2}\left(A k^{\prime} G L^{2}+m^{2} \pi^{2} k^{\prime} G I+m^{2} \pi^{2} E I\right)}
$$

In Ref. [27], Elishakoff and Livshits proposed that the fourth order derivative with respect to time $\left(\rho^{2} I / k^{\prime} G\right) \partial^{4} v / \partial t^{4}$ in Eq. (29) that leads to the term neglected by Timoshenko, be eliminated ab initio. We utilize this idea for the simplified analysis of free vibration of double-walled carbon nanotubes (DWCNTs).

\subsection{DWCNT modeled on the base of simplified Bresse-Timoshenko equations}

Consider the DWCNT. The parameters of the inner tube will be associated with the index " $i$ ", whereas the parameter of the outer tube will be attached an index " $O$ ". The corresponding governing differential equations for the inner tube read

$$
\begin{aligned}
& -k^{\prime} A_{i} G\left(\frac{\partial \psi_{i}}{\partial x}-\frac{\partial^{2} v_{i}}{\partial x^{2}}\right)+p=\rho A_{i} \frac{\partial^{2} v_{i}}{\partial t^{2}} \\
& E I_{i} \frac{\partial^{2} \psi_{i}}{\partial x^{2}}-k^{\prime} A_{i} G\left(\psi_{i}-\frac{\partial v_{i}}{\partial x}\right)=\rho I_{i} \frac{\partial^{2} \psi_{i}}{\partial t^{2}}
\end{aligned}
$$

where $p(x)$ is the van der Waals interaction pressure between the inner and outer tubes. The governing differential equation for the outer tube is

$$
-k^{\prime} A_{o} G\left(\frac{\partial \psi_{o}}{\partial x}-\frac{\partial^{2} v_{o}}{\partial x^{2}}\right)-p=\rho A_{o} \frac{\partial^{2} v_{o}}{\partial t^{2}}
$$




$$
E I_{o} \frac{\partial^{2} \psi_{o}}{\partial x^{2}}-k^{\prime} A_{o} G\left(\psi_{o}-\frac{\partial v_{o}}{\partial x}\right)=\rho I_{o} \frac{\partial^{2} \psi_{o}}{\partial t^{2}}
$$

We eliminate $\psi$ from Eqs (35) and (36) by first solving Eq. (35) for $\partial \psi_{o} / \partial x$; the result is substituted into Eq. (36) after the latter is differentiated with respect of $x$. A resolving governing differential equation for the inner tube reads

$$
E I_{i} \frac{\partial^{4} v_{i}}{\partial x^{4}}+\rho A_{i} \frac{\partial^{2} v_{i}}{\partial t^{2}}-\rho I_{i}\left(1+\frac{E}{k^{\prime} G}\right) \frac{\partial^{2} v_{i}}{\partial x^{2} \partial t^{2}}+\frac{\rho^{2} I_{i}}{k^{\prime} G} \frac{\partial^{4} v_{i}}{\partial t^{4}}+\frac{E I_{i}}{k^{\prime} A_{i} G} \frac{\partial^{2} p}{\partial x^{2}}-\frac{\rho I_{i}}{k^{\prime} A_{i} G} \frac{\partial^{2} p}{\partial t^{2}}-p=0
$$

whereas its counterpart for the outer tube is

$$
E I_{o} \frac{\partial^{4} v_{o}}{\partial x^{4}}+\rho A_{o} \frac{\partial^{2} v_{o}}{\partial t^{2}}-\rho I_{o}\left(1+\frac{E}{k^{\prime} G}\right) \frac{\partial^{2} v_{o}}{\partial x^{2} \partial t^{2}}+\frac{\rho^{2} I_{o}}{k^{\prime} G} \frac{\partial^{4} v_{o}}{\partial t^{4}}-\frac{E I_{o}}{k^{\prime} A_{o} G} \frac{\partial^{2} p}{\partial x^{2}}+\frac{\rho I_{o}}{k^{\prime} A_{o} G} \frac{\partial^{2} p}{\partial t^{2}}+p=0
$$

Following Timoshenko [25,26] and Elishakoff and Livshits [27] we simplify the governing differential equations by neglecting the terms containing the fourth order time derivative to yield:

$$
E I_{i} \frac{\partial^{4} v_{i}}{\partial x^{4}}+\rho A_{i} \frac{\partial^{2} v_{i}}{\partial t^{2}}-\rho I_{i}\left(1+\frac{E}{k^{\prime} G}\right) \frac{\partial^{2} v_{i}}{\partial x^{2} \partial t^{2}}+\frac{E I_{i}}{k^{\prime} A_{i} G} \frac{\partial^{2} p}{\partial x^{2}}-\frac{\rho I_{i}}{k^{\prime} A_{i} G} \frac{\partial^{2} p}{\partial t^{2}}-p=0
$$

for inner tube, and

$$
E I_{o} \frac{\partial^{4} v_{o}}{\partial x^{4}}+\rho A_{o} \frac{\partial^{2} v_{o}}{\partial t^{2}}-\rho I_{o}\left(1+\frac{E}{k^{\prime} G}\right) \frac{\partial^{2} v_{o}}{\partial x^{2} \partial t^{2}}-\frac{E I_{o}}{k^{\prime} A_{o} G} \frac{\partial^{2} p}{\partial x^{2}}+\frac{\rho I_{o}}{k^{\prime} A_{o} G} \frac{\partial^{2} p}{\partial t^{2}}+p=0
$$

for outer tube. For simply supported DWCNTs we take the following expressions for the mode shapes:

$$
\begin{aligned}
& v_{1}(x, t)=Y_{1} \sin \frac{j \pi x}{L} \sin \omega t \\
& v_{2}(x, t)=Y_{2} \sin \frac{j \pi x}{L} \sin \omega t
\end{aligned}
$$

The van der Waals [7] force is taken as proportional to relative transverse displacement $v_{2}(x)-v_{1}(x)$ :

$$
p(x)=c\left[v_{2}(x)-v_{1}(x)\right]
$$

where $c$ is the van der Waals interlayer interaction coefficient and can be estimated as follows

$$
c=\frac{200 \times\left(2 R_{i}\right)}{0.16 D^{2}}, D=0.142 \mathrm{~nm}
$$

Substituting Eqs (43)-(45) into Eqs (41) and (42) and demanding the non-triviality for $Y_{1}^{2}+Y_{2}^{2}$ we get the following determinantal equation:

$$
\operatorname{det}[A]=0
$$

where the elements $a_{\alpha \beta}(\alpha, \beta=1,2)$ of the matrix $A$ are

$$
\begin{aligned}
& a_{11}=E I_{i}\left(\frac{m \pi}{L}\right)^{4}-\rho A_{i} \omega^{2}-\rho I_{i}\left(1+\frac{E}{k^{\prime} G}\right)\left(\frac{m \pi}{L}\right)^{2} \omega^{2}+\frac{E I_{i} c}{k^{\prime} G A_{i}}\left(\frac{m \pi}{L}\right)^{2}-\frac{\rho I_{i} c}{k^{\prime} G A_{i}} \omega^{2}+c \\
& a_{12}=-\frac{E I_{i} c}{k^{\prime} G A_{i}}\left(\frac{m \pi}{L}\right)^{2}+\frac{\rho I_{i} c}{k^{\prime} G A_{i}} \omega^{2}-c \\
& a_{21}=-\frac{E I_{o} c}{k^{\prime} G A_{o}}\left(\frac{m \pi}{L}\right)^{2}+\frac{\rho I_{o} c}{k^{\prime} G A_{o}} \omega^{2}-c \\
& a_{22}=E I_{o}\left(\frac{m \pi}{L}\right)^{4}-\rho A_{o} \omega^{2}-\rho I_{o}\left(1+\frac{E}{k^{\prime} G}\right)\left(\frac{m \pi}{L}\right)^{2} \omega^{2}+\frac{E I_{o} c}{k^{\prime} G A_{o}}\left(\frac{m \pi}{L}\right)^{2}-\frac{\rho I_{o} c}{k^{\prime} G A_{o}} \omega^{2}+c
\end{aligned}
$$


Table 2

Fundamental natural frequency estimates based upon Bernoulli-Euler and BresseTimoshenko theories

\begin{tabular}{cccc}
\hline$L / D_{o}$ & $\begin{array}{c}\omega_{1,1}\left(10^{12} \mathrm{rad} / \mathrm{sec}\right), \\
\text { Bernoulli-Euler Theory }\end{array}$ & $\begin{array}{c}\omega_{1,1}\left(10^{12} \mathrm{rad} / \mathrm{sec}\right), \text { Simplified } \\
\text { Bresse-Timoshenko Theory }\end{array}$ & $\begin{array}{c}\text { Percentagewise } \\
\text { Difference }\end{array}$ \\
\hline 5 & 1.8595 & 1.7054 & 9.0360 \\
6 & 1.2951 & 1.2174 & 4.5561 \\
7 & 0.9526 & 0.9096 & 2.5214 \\
8 & 0.7298 & 0.7042 & 1.5011 \\
9 & 0.5768 & 0.5606 & 0.9499 \\
10 & 0.4673 & 0.4566 & 0.6274 \\
11 & 0.3862 & 0.3789 & 0.4281 \\
12 & 0.3246 & 0.3194 & 0.3049 \\
13 & 0.2766 & 0.2728 & 0.2228 \\
14 & 0.2385 & 0.2356 & 0.1700 \\
15 & 0.2077 & 0.2056 & 0.1231 \\
16 & 0.1826 & 0.1809 & 0.0997 \\
17 & 0.1617 & 0.1604 & 0.0762 \\
18 & 0.1443 & 0.1432 & 0.0645 \\
19 & 0.1295 & 0.1286 & 0.0528 \\
20 & 0.1169 & 0.1162 & 0.0410 \\
\hline
\end{tabular}

\subsection{Numerical results}

For numerical analysis we fix the data at following values following Yoon et al. [7]: inner radius $R_{i}=0.35 \mathrm{~nm}$, outer radius $R_{o}=0.7 \mathrm{~nm}$, thickness $\delta_{0}=0.34 \mathrm{~nm}, E=1 \mathrm{TPa}, G=0.4 \mathrm{TPa}, k^{\prime}=0.8, c=43.39 \mathrm{GPa}, \rho=$ $2.3 \mathrm{~g} / \mathrm{cm}^{3}$.

Table 2 lists the fundamental natural frequency for various values of the length over the outer diameter $L / D$ o evaluated within the Bernoulli-Euler and simplified Bresse-Timoshenko theories, respectively. Table also lists the percentagewise differences between the predictions

$$
\varepsilon=\frac{\left|\omega_{1, m}^{B-E}-\omega_{1, m}^{B-T}\right|}{\omega_{1, m}^{B-T}} \times 100 \%
$$

where $\omega_{1, m}^{B-E}$ is the $m^{\text {th }}$ natural frequency obtained by analysis of DWCNT based on Bernoulli-Euler (B-E) theory; $\omega_{1, m}^{B-T}$ is the $m^{t h}$ natural frequency in the first series of the Bresse-Timoshenko (B-T) theory. For the value $L / D D_{o}=5$ the percentagewise difference is 9 percent; the difference constitutes about 0.6 percent for $L / D_{o}=10$ and decreases to just 0.04 percent for $L / D_{o}=20$. We conclude that for long DWCNTs use of Bresse-Timoshenko theory is not warranted; in these circumstances one can safely use the Bernoulli-Euler theory. However, for short DWCNTs the difference in the estimations provided by two theories can be significant. Hence, the Bresse-Timoshenko theory should be applied for $L / D_{o} \leqslant 10$.

Analogous results pertaining to the second natural frequency are listed in Table 3. Here, the difference between two theories reaches $0.2 \%$ for $L / D_{o}=20$; with decrease of the $L / D_{o}$ ratio the percentagewise difference increases; it constitutes $2.87 \%$ for $L / D_{o}=10$, while it rises up to nearly $25 \%$ for $L / D_{o}=5$.

Table 4 is associated with the third natural frequency. Here, even for long DWCNTs the percentagewise difference is of order of $0.6 \%$; however, for $L / D_{o}=10$, the difference is $6.8 \%$, whereas for $L / D_{o}=5$, the difference constitutes about $30 \%$.

We conclude that in the vibration problems where only the fundamental natural frequency is excited, one has to resort to the refined Bresse-Timoshenko theory for very short DWCNTs. However, if in the vibration problem at hand, first two frequencies play a significant role, then the Bresse-Timoshenko theory is needed already for $L / D$ o around 15 or less. However, if the first three natural frequencies are excited, then even for long carbon nanotubes, with $L / D_{o} \leqslant 20$, one should use Bresse-Timoshenko theory. For further results one can consult the study in Ref. 29.

It must be emphasized that the continuum mechanics modeling is not the only "game in town". Among other approaches the molecular dynamics approach is most prevailing. Ranges of applicability of the continuum models are discussed by Harik [29] and Sears and Batra [30], among others. 
Table 3

Estimates in the second natural frequency based on Bernoulli-Euler and BresseTimoshenko theories

\begin{tabular}{cccc}
\hline$L / D_{o}$ & $\begin{array}{c}\omega_{1,2}\left(10^{12} \mathrm{rad} / \mathrm{sec}\right), \\
\text { Bernoulli-Euler Theory }\end{array}$ & $\begin{array}{c}\omega_{1,2}\left(10^{12} \mathrm{rad} / \mathrm{sec}\right), \text { Simplified } \\
\text { Bresse-Timoshenko Theory }\end{array}$ & $\begin{array}{c}\text { Percentagewise } \\
\text { Difference }\end{array}$ \\
\hline 5 & 6.7209 & 5.3629 & 25.3221 \\
6 & 4.9499 & 4.0801 & 16.2188 \\
7 & 3.7236 & 3.1818 & 10.1027 \\
8 & 2.8813 & 2.5366 & 6.4275 \\
9 & 2.2889 & 2.0621 & 4.2291 \\
10 & 1.8595 & 1.7054 & 2.8734 \\
11 & 1.5395 & 1.4316 & 2.0120 \\
12 & 1.2951 & 1.2174 & 1.4488 \\
13 & 1.1043 & 1.0471 & 1.0666 \\
14 & 0.9526 & 0.9096 & 0.8018 \\
15 & 0.8301 & 0.7972 & 0.6135 \\
16 & 0.7298 & 0.7042 & 0.4774 \\
17 & 0.6466 & 0.6264 & 0.3767 \\
18 & 0.5768 & 0.5606 & 0.3021 \\
19 & 0.5177 & 0.5047 & 0.2424 \\
20 & 0.4673 & 0.4566 & 0.1995 \\
\hline
\end{tabular}

Table 4

Estimates in the third natural frequency based on Bernoulli-Euler and BresseTimoshenko theories

\begin{tabular}{cccc}
\hline$L / D_{o}$ & $\begin{array}{c}\omega_{1,3}\left(10^{12} \mathrm{rad} / \mathrm{sec}\right), \\
\text { Bernoulli-Euler Theory }\end{array}$ & $\begin{array}{c}\omega_{1,3}\left(10^{12} \mathrm{rad} / \mathrm{sec}\right), \text { Simplified } \\
\text { Bresse-Timoshenko Theory }\end{array}$ & $\begin{array}{c}\text { Percentagewise } \\
\text { Difference }\end{array}$ \\
\hline 5 & 12.084 & 9.3188 & 29.6733 \\
6 & 9.272 & 7.2991 & 21.1712 \\
7 & 7.458 & 5.9135 & 16.5740 \\
8 & 6.062 & 4.8810 & 12.6733 \\
9 & 4.950 & 4.0801 & 9.3349 \\
10 & 4.082 & 3.4479 & 6.8045 \\
11 & 3.408 & 2.9435 & 4.9845 \\
12 & 2.881 & 2.5366 & 3.6958 \\
13 & 2.465 & 2.2051 & 2.7890 \\
14 & 2.131 & 1.9322 & 2.1333 \\
15 & 1.860 & 1.7054 & 1.6590 \\
16 & 1.636 & 1.5152 & 1.2963 \\
17 & 1.451 & 1.3544 & 1.0366 \\
18 & 1.295 & 1.2174 & 0.8327 \\
19 & 1.163 & 1.0997 & 0.6793 \\
20 & 1.050 & 0.9981 & 0.5569 \\
\hline
\end{tabular}

The applications of the continuum models appear in need of reevaluation in light of the recent article by Gupta, Bosco and Batra [31]. Specifically, they studied the free vibrations of zigzag single-wall carbon nanotubes of aspect ratio (length/diameter) $\sim 6$. They founds that the frequencies of the inextensional modes of the continuum structures deviate noticeably from those deviations increasing with an increase in the circumferential wave number.

At this juncture the reader may inquire, and justifiably so, about the nonlinear problems in structural dynamics. This section is included here in order to elucidate some novel analyses of nonlinear stochastic problems. It is hoped that these nonlinear treatments will find their way in the nanomechanics applications.

\section{Eighty one combinations of the stochastic linearization criteria by the moment approach}

In this section we follow closely the paper [32] Booton [33] and Kazakov [34] introduced the stochastic linearization method nearly simultaneously in 1953 and 1954, respectively. In nearly half a century since its inception, many review articles have been written, and its description appears in specialized monographs, that include the treatment of non-linear random vibration. In these two works, Booton [33] and Kazakov [34] replace the non-linear dynamic 
system by the linear system that is equivalent to the original, non-linear one in some probabilistic sense. The criterion that was suggested is that of the minimum mean-square deviation between the original non-linear expression of the force $\varphi(X)$, where $X$ is the displacement, and the linear counterpart $k_{e q} X$, where $k_{e q}$ is the spring constant of the equivalent linear system. The aim is formulated as follows: given the probabilistic properties of the excitation $F(t)$, find the mean-square values of the displacement $E\left(X^{2}\right)$ and the velocity $E\left(\dot{X}^{2}\right)$. Booton [33] and Kazakov [34], as well as numerous other investigators (in our estimate over 400) - utilize the following formula for the equivalent spring constant:

$$
k_{e q}^{(1)}=E[X \varphi(X)] / E\left(X^{2}\right)
$$

however, Kazakov's [30] classical work contains another criterion too. It demands that the mean-square values of the non-linear force $E\left[f^{2}(X)\right]$ and its replacement $E\left[\left(k_{e q} X\right)^{2}\right]$ be equal:

$$
E\left[\varphi^{2}(X)\right]=E\left[\left(k_{e q} X\right)^{2}\right]
$$

resulting the following expression of $k_{e q}$ :

$$
k_{e q}^{(2)}=\sqrt{E\left[\varphi^{2}(X)\right] / E\left(X^{2}\right)}
$$

Unfortunately, investigators almost uniformly do not report this classic result of Kazakov's [33] work. This reminds us of Mark Twain's [35] definition of the classical work: "A classic is something that everybody wants to have but nobody wants to read." In this study, a short summary is given of some recent work on stochastic linearization criteria. Equation (50) will be obtained by means not utilized by either the Booton [33] or Kazakov [34] derivations. Likewise, some energy criteria, proposed in the eighties, will be re-derived. In total, 81 criteria will be discussed, along with the possible implications and significance of such a multiplicity.

\subsection{Basic equations}

Consider a single degree of freedom system described by the following non-linear differential equation:

$$
m \ddot{X}+\varphi(X)+\psi(\dot{X})=F(t)
$$

where $\varphi(X)$ is the non-linear restoring force, while $\psi(\dot{X})$ is the non-linear damping force, m denotes the mass: $F(t)$ is the excitation with specified probabilistic properties. We will utilize the method of moments as suggested by Yamada [36-38] and Fujita [39] for the deterministic problems (see also Finlayson [40]). As Finlayson [40] notes, "For the ordinary differential equations governing. . . phenomenon the weighting functions are $x, x^{2}, x^{3}, \ldots$ Thus successively higher moments of the residual are required to be zero." We replace the non-linear restoring force $\varphi(x)$ by the linear equivalent $k_{e q} X$. Since these two quantities are unequal, unless the restoring force is linear, we form the deficiency, or error

$$
\varepsilon_{\varphi}(X)=\varphi(X)-k_{e q} X
$$

We demand that the deficiency be probabilistically orthogonal to $x$, i.e

$$
\left\langle\varepsilon_{\varphi}(X), X\right\rangle=0
$$

where the angle brackets denote the inner product

$$
\left\langle f_{1}(X), f_{2}(X)\right\rangle=E\left[f_{1}(X) f_{2}(X)\right]=\int_{-\infty}^{\infty} f_{1}(x) f_{2}(x) p_{X}(x) d x
$$

where $p_{X}(x)$ is the probability density function of $x$. Equation (55) leads to the requirement

$$
\left\langle\varepsilon_{\varphi}(X), X\right\rangle=\left\langle\varphi(X)-k_{e q} X, X\right\rangle=0
$$

from which we find $k_{e q}$

$$
k_{e q}=\frac{\langle\varphi(X), X\rangle}{\langle X, X\rangle}
$$


Bearing in mind the definition of the inner product in Eq. (56), we conclude that Eq. (58) coincides with Eq. (50); thus $k_{e q}$ in Eq. (57) is $k_{e q}^{(1)}$ :

$$
\left\langle\varepsilon_{\varphi}(X), X\right\rangle=\left\langle\varphi(X)-k_{e q}^{(1)} X, X\right\rangle=0
$$

The expression for $c_{e q}$ can be obtained by forming the deficiency or error $\varepsilon_{\psi}(\dot{X})$, between the damping force $\psi(\dot{X})$ and its linear replacement $c_{e q} \dot{X}$

$$
\varepsilon_{\psi}(\dot{X})=\psi(\dot{X})-c_{e q} \dot{X} .
$$

According to the method of moments [36-40], we require the following inner product to vanish:

$$
\left\langle\varepsilon_{\psi}(\dot{X}), \dot{X}\right\rangle=0
$$

or

$$
\left\langle\psi(\dot{X})-c_{e q} \dot{X}, \dot{X}\right\rangle=\langle\psi(\dot{X}), \dot{X}\rangle-c_{e q}\langle\dot{X}, \dot{X}\rangle=0
$$

Thus,

$$
c_{e q}=\langle\psi(\dot{X}), \dot{X}\rangle /\langle\dot{X}, \dot{X}\rangle=c_{e q}^{(1)}
$$

which coincides with the expression used by the pioneers of the stochastic linearization.

Thus, Eqs (50) and (59) can be derived without utilization of the so-called minimum mean-square difference criterion, as it was done in the literature. The derivation via the method of moments is much simpler than the explanation utilized in the literature. The demand that the mean-square values of the non-linear and linear dissipation forces to be equal, i.e. that

$$
E\left[\psi^{2}(\dot{X})\right]=E\left[\left(c_{e q} \dot{X}\right)^{2}\right]
$$

yields the following expression for $c_{e q}$ :

$$
c_{e q}^{(2)}=\sqrt{E\left[\psi^{2}(\dot{X})\right] / E\left(\dot{X}^{2}\right) E\left(\dot{X}^{2}\right)}
$$

\subsection{Energy linearization of stiffness}

Wang and Zhang [41], Elishakoff and Zhang [42], Zhang, Elshakoff and Zhang [43] (see also the recent study of Murayov, Turner, Robinson and Rizzi [44]) utilized a potential energy of the system as a parameter with respect to which the linearization should be performed. This criterion is derived here in much simpler manner as follows. The potential energy of deformation stored in the non-linear spring equals

$$
P(X)=\int_{0}^{X} \varphi(x) d x
$$

the potential energy of the associated equivalent linear spring is

$$
P_{e q}(X)=k_{e q} X^{2} / 2
$$

We form the deficiency with respect to energy $\varepsilon_{p}$, as follows:

$$
\varepsilon_{p}(X)=P(X)-k_{e q} X^{2} / 2
$$

and demand it to be orthogonal to $X^{2}$ :

$$
\left\langle\varepsilon_{p}(X), X^{2}\right\rangle=0
$$

or

$$
\left\langle P(X), X^{2}\right\rangle-k_{e q}\left\langle X^{2} / 2, X^{2}\right\rangle=0
$$


leading to the following expression for $k_{e q}=k_{e q}^{(3)}$ :

$$
k_{e q}^{(3)}=2 E\left[X^{2} P(X)\right] / E\left(X^{4}\right)
$$

Another criterion based on the potential energy is somewhat analogous to that by Kazakov [34]. It demands that the mean-square value of the potential energy of the original system $E\left[P^{2}(X)\right]$ has to coincide with its counterpart in the linear system, namely

$$
E\left[P^{2}(X)\right]=E\left[\left(k_{e q} X^{2} / 2\right)^{2}\right]
$$

The attendant expression for the equivalent spring is

$$
k_{e q}^{(4)}=2 \sqrt{E\left[P^{2}(X)\right] / E\left(X^{4}\right)}
$$

Elishakoff and Bert [45] proposed a criterion based on the concept of the complementary energy. Namely, the complementary energy $C(X)=X \varphi(X)-P(X)$ is used as the basis for linearization.

The deficiency between the complementary energy $C(X)$ of the original nonlinear system and that of the linear system $k_{e q} X^{2} / 2$,

$$
\varepsilon_{c}(X)=C(X)-k_{e q} X^{2} / 2
$$

is being first formed. Then it is made orthogonal to $X^{2}$ to yield

$$
k_{e q}^{(5)}=2 E\left[X^{2} C(X)\right] / E\left(X^{2}\right)
$$

An additional criterion in terms of the complementary energy can be suggested, that of equality of the mean-square values of $C(X)$ and its linear counterpart

$$
E\left[C^{2}(X)\right]=E\left(k_{e q} X^{2} / 2\right)^{2}
$$

An attendant expression for the equivalent stiffness is

$$
k_{e q}^{(6)}=2 \sqrt{E\left[C^{2}(X)\right] / E\left(X^{4}\right)}
$$

It appears instructive to turn now to the linearization in the damping element $\psi(\dot{X})$ in Eq. (53).

\subsection{Dissipation-energy linearization of damping}

Equation (63) gave a derivation of the linearized damping coefficient $c_{e q}$ in a manner different from its conventional derivation. However, other expressions can be obtained. In this context the energy dissipation function

$$
D(\dot{X})=\int_{0}^{\dot{X}} \psi(z) d z
$$

is instructive. This concept was utilized by Wang and Zhang [41] in their study. We will derive their expression by other means. We form the residual $\varepsilon_{D}$ stemming due to the replacement of the damping force $c_{e q} \dot{X}$. We demand the deficiency

$$
\varepsilon_{D}=D(\dot{X})-c_{e q} \dot{X}^{2} / 2
$$

between the energy dissipation functions representing the non-linear and linear systems to be orthogonal to $\dot{X}^{2}$,

$$
\left\langle\varepsilon_{D}, \dot{X}^{2}\right\rangle=0
$$

The associated expression for $c_{e q}$ reads

$$
c_{e q}^{(3)}=2 E\left[\dot{X}^{2} D(\dot{X})\right] / E\left(\dot{X}^{4}\right)
$$


One can also demand equality of the mean-square values of the appropriate, linear and non-linear, energy dissipation functions (see Elishakoff [46])

$$
E\left[D^{2}\right]=E\left[\left(c_{e q} \dot{X}^{2} / 2\right)^{2}\right]
$$

From the condition we obtain

$$
c_{e q}^{(4)}=2 \sqrt{E\left[D^{2}\right] / E\left(\dot{X}^{4}\right)}
$$

One can also formally introduce the "complementary energy dissipation function":

$$
M(\dot{X})=\dot{X} \psi(\dot{X})-D(\dot{X})
$$

With this concept we can arrive at two criteria. One demands that the deficiency to be orthogonal to $\dot{X}^{2}$, yielding:

$$
\varepsilon_{M}=M(\dot{X})-c_{e q} \dot{X}^{2} / 2
$$

be probabilistically orthogonal to $\dot{X}^{2}$. The attendant expression for $c_{e q}$ is:

$$
c_{e q}^{(5)}=2 E\left[\dot{X}^{2} M(\dot{X})\right] / E\left(\dot{X}^{4}\right)
$$

On the other hand, demand that

$$
E\left[M^{2}(\dot{X})\right]=E\left[\left(c_{e q} \dot{X}^{2} / 2\right)\right]
$$

leads to

$$
c_{e q}^{(6)}=2 \sqrt{E\left[M^{2}(\dot{X})\right] / E\left(\dot{X}^{4}\right)}
$$

Thus, we have six different expressions for the equivalent spring constant and six different expressions for the equivalent damping coefficients. Yet, these do not exhaust all possible linearization avenues.

In the process of evaluating the expressions for the equivalent stiffness and damping, it may appear that we have overlooked the goal of the analysis itself! This evaluation of the mathematical expectations involved in the expressions for $k_{e q}$ and $c_{e q}$ presupposes the knowledge of the probability density involved, namely $p_{X}(x)$. Yet, had we known the density we would not go through the trouble of utilizing the linearization technique, but would directly evaluate the desired response quantities

$$
E\left(X^{2}\right)=\int_{-\infty}^{\infty} x p_{X}(x) d x, \quad E\left(\dot{X}^{2}\right)=\int_{-\infty}^{\infty} \dot{x}^{2} p_{\dot{X}}(\dot{x}) d x
$$

Yet, we do not know $p_{X}(x)$ and $p_{\dot{X}}(\dot{x})$. If so, the formulas for $k_{e q}$ and $c_{e q}$ may appear to be void of sense. Indeed, to evaluate $c_{e q}$ we need to know the density, which is not known to us. This situation is not entirely different from the use of the Rayleigh's quotient in vibration analysis. For the Bernoulli-Euler beams it reads

$$
\omega^{2}=\frac{\int_{0}^{L} E I\left(d^{2} W / d x^{2}\right)^{2} d x}{\int_{0}^{L} \rho(x) A(x) W^{2}(x) d x}
$$

where $\omega$ is the natural frequency to be determined, $E(x)=$ modulus of elasticity, $I(x)=$ moment of inertia of the beam's cross-section, $\rho(x)=$ material density, $A(x)=$ cross-sectional area, $W(x)=$ mode shape. As Professor V. V. Bolotin remarked during the exposition of this subject, the Rayleigh's quotient maintains, in essence, that in order to obtain the natural frequency, one needs the knowledge of the mode shape; yet, if we were in possession of the mode shape, we would not need the Rayleigh quotient, but obtain the natural frequency from the governing differential equation itself, 


$$
\frac{d^{2}}{d x^{2}}\left[E(x) I(x) \frac{d^{2} W(x)}{d x^{2}}\right]=\rho(x) A(x) \omega^{2} W(x)
$$

as

$$
\omega^{2}=\frac{d^{2}}{d x^{2}}\left[E(x) I(x) \frac{d^{2} W(x)}{d x^{2}}\right] / \rho(x) A(x) W(x)
$$

The main idea by Rayleigh was to use not an exact, but an approximate expression for the mode shape. We substitute the expression $\tilde{W}(x)$ that approximates the true (and unknown) mode shape. The quotient in Eq. (90) yields an approximate expression for the natural frequency.

An analogous idea is utilized to justify the expressions we obtained for the linearization coefficients. We approximate the true (and unknown) probability density $p_{X}(x)$ by density $\tilde{p}_{X}(x)$ that contains some unknown parameter(s). Consider as an example an approximation that has unspecified parameters $\tilde{\sigma}_{X}$ and $\tilde{\sigma}_{\dot{X}}$, these quantities being mean-square deviations of the approximating process and its derivative, respectively. Mathematical expectation involved in determination of the stiffness and damping coefficients become dependent upon $\tilde{\sigma}_{X}$ and $\tilde{\sigma}_{\dot{X}}$. Thus,

$$
k_{e q}=k_{e q}\left(\tilde{\sigma}_{X}\right), \quad c_{e q}=c_{e q}\left(\tilde{\sigma}_{\dot{X}}\right)
$$

At this juncture we pretend we know $k_{e q}$ and $c_{e q}$. These characterize the replacing linear system governed by the differential equation

$$
m \ddot{X}+k_{e q}\left(\tilde{\sigma}_{X}\right) X+c_{e q}\left(\tilde{\sigma}_{\dot{X}}\right) \dot{X}=F(t)
$$

well studied in the random vibration literature. For example, if $F(t)$ is a random process that is stationary in the wide sense, than the spectral analysis yields the mean-square responses. If, for example, the excitation is the white noise, and mathematical expectation is zero, i.e. its spectral density is constant, so we get

$$
E\left(X^{2}\right)=\frac{\pi S_{0}}{c_{e q} k_{e q}}, E\left(\dot{X}^{2}\right)=\frac{\pi S_{0}}{m c_{e q}}
$$

Yet, $E\left(X^{2}\right)$ due to utilized approximation $p_{X}(x) \approx \tilde{p}_{X}(x)$ equals $\tilde{\sigma}_{X}^{2}$, while, on the other hand $k_{e q}$ is a function of $\tilde{\sigma}_{X}$. Likewise, $E\left(\dot{X}^{2}\right)$ equals $\tilde{\sigma}_{\dot{X}}^{2}$, whereas $c_{e q}$ is a function of $\tilde{\sigma}_{\dot{X}}$. Thus, Eqs (94) and (95) can be rewritten as

$$
\tilde{\sigma}_{X}^{2}=\pi S_{0} / c_{e q} k_{e q}\left(\tilde{\sigma}_{X}\right), \quad \tilde{\sigma}_{\dot{X}}^{2}=\pi S_{0} / m c_{e q}\left(\tilde{\sigma}_{\dot{X}}\right)
$$

yielding equations for $\tilde{\sigma}_{X}$ and $\tilde{\sigma}_{\dot{X}}$. On this stage the approximate density is fully revealed, for the control parameter is determined; likewise, our initial problem, which was more modest than that of finding the probability density, is solved, for $E\left(X^{2}\right)$ and $E\left(\dot{X}^{2}\right)$ are approximated by $\tilde{\sigma}_{X}^{2}$ and $\tilde{\sigma}_{\dot{X}}^{2}$, respectively, and determined from Eq. (96).

Analogously, one can show that criteria based upon approximating probability density can be suggested. For further details the interested reader may consult Ref. 28.

\subsection{Why do we need so many criteria?}

One can arrive [32] at nine different criteria for evaluating the equivalent stiffness $k_{e q}$ and 9 different conditions for evaluating the stiffness coefficient $c_{e q}$. Since each criterion for computing $k_{e q}$ can be combined with any criterion for calculating $c_{e q}$, we conclude that we arrive at $9 \times 9=81$ different criteria for solving the nonlinear stochastic problem at hand.

The multiplicity of the methods to arrive at the sought solution may appear to be alarming in several respects.

The first question that begs itself to be asked is "Why do we need so many criteria? Why not use a single criterion?" The reply to this question may be a question itself: "Why not?" Indeed, for solving linear deterministic problems there are a multiplicity of methods. For example, for solving the linear eigenvalue problem one can resort to the methods of numerical integration method, method of successive approximations, Rayleigh-Ritz method, BubnovGalerkin method, Petrov-Galerkin method, finite difference method, finite element method, etc. An even more direct connection exists between the problem at hand and the failure criteria in the mechanics of solids. We have criteria of maximum stress, maximum strain, St-Venant's criterion, Tresca criterion, Goldenblat-Kopnov criterion, Tsai-Wu 
criterion, etc. It appears that while the method of exact solution of nonlinear stochastic differential equations is absent a tolerance may be exercised toward various stochastic linearization criteria.

How to choose the stochastic linearization criterion from their multiplicity? Not unlike the failure criteria, accumulation of the experience appears to be useful, for in various circumstances different criteria may prove to be advantageous over the others.

It appears instructive to classify 81 methods in two separate classes: like criteria and unlike criteria. Like criteria are the ones with analogous reasoning both for evaluating $k_{e q}^{(\alpha)}$ and $c_{e q}^{(\beta)}$. For like criteria, we have $\alpha=\beta$. Thus we have 9 like criteria. For example probabilistic orthogonality criteria of the restoring force difference and the damping force difference constitute one pair of the like criteria with $\alpha=\beta=1$. These are classical criteria. The rest 72 criteria constitute the unlike ones: for example, criterion of probabilistic orthogonality for the force difference for evaluating $k_{e q}$, and the criterion of equal mean-squares of the energy dissipation functions correspond to $\alpha=1, \beta=2$. The establishment of the class of problems in which any of 80 non-classical criteria is advantageous over the classical one appears to be of interest. Selective review of the method is given in Ref. 43. Elsihakoff and Colajanni [47-50] and Socha and Pawleta [51] reexamined the very foundations of the stochastic linearization method. There is no consensus on this issue. No additional comments will be made in this study having in mind to return to this issue at length.

\subsection{What to do with multiplicity of criteria?}

A natural question arises: What to do with these criteria? Popov and Paltov [52], when discussing two criteria suggested by Kazakov [34], came up with the recommendation to use the arithmetic mean of results yielded by two methods, as a better approximation for the mean-square response, to bracket the exact result. Bolotin [53] showed that such an approach was not justified, since in the particular problem, studied by him, the exact solution was not backed by the results obtained by two competitive criteria. We will refrain therefore to recommend to solve every problem by 81 competitive means and then to average the results. Rather, a study may be recommended to establish the regions when a specific criterion can be advantageous, over the other criteria. It appears that one can construct arbitrary number of stochastic linearization criteria, for approximate solution of stochastic response problems. In such circumstances the importance of closed-form benchmark solutions, on one hand, and fully numerical, Monte Carlo solutions, cannot be overestimated [54]. The methods described above can be combined with recent methodology developed by Michlin and Grabarnik [55]. Most recent efforts on energy-based linearization criteria include papers by Elishakoff et al. [56], and Elishakoff and Andiamasy [57]. Extensive and up-to-date exposition of stochastic linearization technique is given in the recent monograph by Socha [58].

\section{Conclusions}

By even a superficial glance of the two topics considered in this paper one can still draw some conclusions of somewhat general nature:

1. It appears that it is not advisable to deal solely with either deterministic analysis or stochastic treatment of the problems. It so happens that the sole involvement in one of these problems may lead to in lack of appreciation of one of these approaches. Since the deterministic analysis is the cornerstone of the stochastic analysis, it appears imperative that the vibration community at large does not abandon the nitty-gritty of the deterministic analysis.

2. It is still under discussion if the continuum models can be satisfactorily applied to the carbon nanotubes. In such circumstances, simple approximations for the vibration frequencies and buckling loads are called for.

3. There has been much progress in the field of stochastic linearization techniques that was dealt by numerous authors over the half a century (Crandall [57], Roberts and Spanos [58] and Magnus and Popp [59]).

4. Multiplicity of choices for stochastic linearization technique allows for the opportunities for the choice of the most appropriate criterion for the problem under consideration.

For the detailed discussion on other future directions in structural engineering dynamics the readers can consult the books edited by Ewins and Inman [59] and Braun et al. [60]. 


\section{Acknowledgements}

We appreciate helpful comments communicated to us by Dr. J. Storch of the Satellite Development Center (Boeing Company), and Mr. K. Dujat of IFMA, France.

\section{References}

[1] D. Qian, G.J. Wagner and W.K. Liu, Mechanics of carbon nanotubes, Applied Mechanics Reviews 55(6) (2002), 495-533.

[2] T.S. Gates and J.A. Hinkley, Computational Materials: Modeling and Simulation of Nanostructured Materials and Systems, NASA Langley Research Center NASA/TM-2003-212163.

[3] W.K. Liu, E.G. Karpov and H.S. Park, Nano Mechanics and Materials: Theory, Multiscale Methods and Applications, John Wiley \& Sons Ltd (England: West Sussex), 2006.

[4] K.Y. Xu, X.N. Guo and C.Q. Ru, 'Vibration of a Double-Walled Carbon Nanotube Aroused by Nonlinear Intertube van der Waals Forces, Journal of Applied Physics 99 (2006), 0643303.

[5] K.Y. Xu, E.C. Aifantis and Y.H. Yan, Vibration of Double-Walled Carbon Nanotube with Different Boundary Conditions between Inner and Outer Tubes, ASME Journal of Applied Mechanics 75 (2008), 021013.

[6] C.Q. Ru, Elastic Models for Carbon Nanotubes, in: Encyclopedia of Nanoscience and Nanotechnology, (Vol. 2), H.S. Nalwa, ed., American Scientific Stevenson Ranch CA, 2004, pp. 731-744.

[7] J. Yoon, C.Q. Ru and A. Mioduchowski, 'Terahertz Vibration of Short Carbon Nanotubes Modeled as Timoshenko Beams, ASME Journal of Applied Mechanics 72 (2005), 10-17.

[8] C.M. Wang, V.B.C. Tan and Y.Y. Zhang, Timoshenko Beam Model for Vibration Analysis of Multi-Walled Carbon Nanotubes, Journal of Sound and Vibration 294 (2006), 1060-1072.

[9] X.Q. He, M. Eisenberger and K.M. Liew, The Effect of van der Waals Interaction Modeling on the Vibration Characteristics of Multiwalled Carbon Nanotubes, Journal of Applied Physics 100 (2006), 124317.

[10] C.Q. Ru, Axially Compressed Buckling of a Doublewalled Carbon Nanotube Embedded in an Elastic Medium, Journal of the Mechanics and Physics of Solids 49 (2001), 1265-1279.

[11] C.Y. Wang, C.Q. Ru and A. Mioduchowski, Axially Compressed Buckling of Pressured Multiwall Carbon Nanotubes, International Journal of Solids and Structures 40 (2002), 3893-3911.

[12] I.G. Bubnov, Reviews of Professors Kirpichev, Belzetskii, Bubnov and Kolosoff on works of Professor Timoshenko, awarded the D. I. Zhuravskii Prize Sbornik C.-Petersburgskogo Instituta Inzhenerov Putei Soobshschenia (Collection of St. Petersburg Institute of Transportation Engineering), 81 (1913) 1-40 (see also Bubnov I G 1956 Selected Works Sudpromgiz Publishers Leningrad 136-139 in Russian).

[13] B.F. Galerkin, Rods and plates. Series in Some Problems of Elastic Equilibrium of Columns and Plates, Vestnik Inzhenerov (Engineering Transactions) 1 (1915), 897-908 (in Russian).

[14] G.I. Petrov, Applications of the Galerkin Method to the Problem on Stability of Viscous Liquid Flow, Prikladnaya Mathematika i Mekhanika (PMM-Applied Mathematics and Mechanics) 4 (1940), 3-11 (in Russian).

[15] I. Elishakoff and D. Pentaras, 'Fundamental Natural Frequencies of Double-Walled Carbon Nanotubes, Journal of Sound and Vibration 322(4-5) (2009), 652-664.

[16] T. Natsuki, Q.-Q. Ni and M. Endo, Analysis of the Vibration Characteristics of Double-Walled Carbon Nanotubes, Carbon 46 (2008), $1570-1573$.

[17] C.Q. Ru, Private Communications, Aug. 8, 2008.

[18] W.J. Duncan, Galerkin's method in mechanics and differential equations, Aeronautical Research Committee Reports and Memoranda No. $1978,1937$.

[19] K. Chang, In Study, Researchers Find Nanotubes May Pose Health Risks Similar to Asbestos, The New York Times, May $21,2008$.

[20] C.A. Poland, R. Duffin, I. Kinloch, A. Maynard, W.A.H. Wallace, A. Seaton, V. Stone, S. Brown, W. MacNee and K. Donaldson, Carbon Nanotubes Introduced Into the Abdominal Cavity of Mice Show Asbestos-Like Pathogenicity in a Pilot Study, Nature Nanotechnology 3 (2008), 423-428, doi:10.1038/nnano.2008.111.

[21] C.M. Wang, V.B.C. Tan and Y.Y. Zhang, Timoshenko Beam Model for Vibration Analysis of Multi-Walled Carbon Nanotubes, Journal of Sound and Vibration 294 (2006), 1060-1072.

[22] J. Yoon, C.Q. Ru and A. Mioduchowski, Timoshenko-Beam Effects on Transverse Wave Propagation in Carbon Nanotubes, Composites Part B: engineering 35 (2004), 87-93.

[23] Y.Y. Zhang, C.M. Wang and V.B.C. Tan, Buckling of Multiwalled Carbon Nanotubes Using Timoshenko Beam Theory, Journal of Engineering Mechanics 132 (2006), 952-958.

[24] W. Weaver, Jr., S.P. Timoshenko and D.H. Young, Vibration Problems in Engineering, (5th edition), John Wiley and Sons, New York, 1990, pp. 434-435.

[25] S.P. Timoshenko, On the Correction for Shear of the Differential Equation for Transverse Vibrations of Prismatic Bars, Philosophical Magazine 41(6), No. 245, (1921), 744-746.

[26] S.P. Timoshenko, The collected papers, New York, McGraw-Hill Book Co., 1953, pp. 288-290.

[27] I. Elishakoff and D. Livshits, Some Closed Form Solutions in Random Vibrations of Timoshenko Beams, Probabilistic Engineering Mechanics 4 (1989), 49-54.

[28] I. Elishakoff and D. Pentaras, Natural Frequencies of Carbon Nanotubes Based on Simplified Bresse-Timoshenko Theory, Journal of Computational and Theoretical Nanoscience 5(7) (2009). 
[29] V.M. Harik, Ranges of Applicability for Continuum Beam Model in the Mechanics of Carbon Nanotubes and Nanorods, Solid State Communications 120 (2001), 331-335.

[30] A. Sears and R.C. Batra, Macro-Mechanics Properties of Carbon Nanotubes from a Molecular Mechanics Simulations, Physical Review, B69 2006, article No. 085410.

[31] S.S. Gupta, F.G. Bosco and R.C. Batra, Breakdown of Structural Models for Vibrations of Single-Wall Zigzag Carbon Nanotubes, 106, 2009, article No. 063527.

[32] I. Elishakoff, Multiple Combination of the Stochastic Linearization Criteria by the Moments Approach, Journal of Sound and Vibration 237(3) (2000), 550-559.

[33] J.R. Booton, The Analysis of Nonlinear Control System with Random Inputs, Proceedings of the Symposium on Nonlinear Circuit Analysis, Politechnic Institute of Brooklyn, 1953, pp. 369-397.

[34] I.E. Kazakov, An approximate method for the statistical investigation for nonlinear systems, Trudy Voenno-Vozdushnoi Akademii imeni Prof. N. E. Zhukovskogo, 1954, pp. 1-52 (in Russian).

[35] J.M. Cohen and M.J. Cohen, The Penguin Dictionary of Quotations, Penguin Books, Harmondsworth, Middesex, 1960, p. 401.

[36] H. Yamada, Rep Res Inst Fluid Eng Kyushu Univ 3 (1947), 29.

[37] H. Yamada, Method of Investigation of Laminar Boundary-Layer Equations, Rep Res Inst Fluid Eng 4 (1948), 27-42 (in Japanese).

[38] H. Yamada, A Method of Approximate Integration of the Laminar Boundary-Layer Equation, Rep Res Inst Fluid Eng 6 (1950), 87-98 (in Japanese).

[39] H. Fujita, On the Problem of Heat Conduction at High Temperature, Mem Coll Agr Kyoto Imp Univ 52 (1951), 31.

[40] B.A. Finlayson, The Method of Weighted Residuals and Variational Principles, Academic Press, New York, 1972.

[41] C. Wang and X.T. Zhang, Technique in Nonlinear Random Vibration, Proceeding of the International Conference on Nonlinear Mechanics, 1985, pp. 959-964.

[42] I. Elishakoff and X.T. Zhang, An Appraisal of Different Stochastic Linearization Criteria, Journal of Sound and Vibration 153 (1992), $370-375$.

[43] X.T. Zhang, I. Elishakoff and R.C. Zhang, A Stochastic Linearization Technique Based on Minimum Mean-Square Deviation of the Potential Energies, in: Stochastic Structural Dynamics- New Theoretical Developments, Y. K. Lin and I. Elishakoff, eds, Springer, Berlin, 1991, pp. 327-338.

[44] A.A. Muravyov, T.L. Turner, J.H. Robinson and S.A. Rizzi, A New Stochastic Equivalent Linearization Implementation for Prediction of Geometrically Nonlinear Vibrations, Paper 99-1376, $40^{\text {th }}$ AIAA/ ASME/ ASCE Structural Dynamics Conference, St. Louis, 1999 pp. 1489-1497.

[45] I. Elishakoff and C.W. Bert, Complementary energy criterion in nonlinear stochastic dynamics, in: Application of Stochastic and Probability, R.L. Melchers and M.G. Stewart, eds, A. A. Balkema, Rotterdam, 1999, pp. 821-825.

[46] I. Elishakoff, Some results in stochastic linearization of nonlinear systems, in: Nonlinear Dynamics and Stochastic Mechanics, W.H. Kliemann and Namachchivaya, eds, CRC Press, Boca Raton 1995, pp. 259-281.

[47] I. Elishakoff and P. Colajanni, Stochastic Linearization Critically Re-Examined, Chaos, Solitons \& Fractals 8 (1997), 1957-1972.

[48] P. Colajanni and I. Elishakoff, A Subtle Error in Conventional Stochastic Linearization Technique, Chaos, Solitons \& Fractals 9 (1998), 479-491.

[49] P. Colajanni and I. Elishakoff, A New Look at the Stochastic Linearization Techniques, Chaos, Solitons \& Fractals 9 (1998), 1611-1623.

[50] I. Elishakoff and E. Colajanni, Booton's Problem Re-Examined, Journal of Sound and Vibration 210 (1998), 683-691.

[51] L. Socha and M. Pawleta, Corrected equivalent linearization of stochastic dynamic systems, Machine Dynamics Problems 7 (1994), 49-161.

[52] E.P. Popov and I.N. Paltov, Approximate Methods of Investigation of Nonlinear Automatic System, Fizmatgiz Publishers, Moscow, 1960 (in Russian).

[53] V.V. Bolotin, Application of the Methods of the Theory of Probability and the Theory of Reliability to Analysis of Structures, State Publishing House for Civil Engineering, Moscow, 1971 (in Russian). [English translation: FTD-MT-24-771-73, Foreign Technology Division, Wright-Patterson AFB, Ohio, 1974].

[54] I. Elishakoff, Stochastic Linearization Technique: A New Interpretation and a Selective Review, The Shock and Vibration Digest 32(3) (2000), 179-188.

[55] Y.H. Michlin and G. Grabarnik, Sequential Testing for Comparison of the Mean Time between Failures for Two Systems, IEEE Transactions on Reliability 56(2) (2007), 321-331.

[56] I. Elishakoff, L. Andriamasy and M. Dolley, Application and Extension of the Stochastic Linearization by Anh and Di Paola, Acta Mechanica 204(1-2) (2009), 89-98.

[57] S.H. Crandall, ed., Random vibration, (volume 2, Technology Press of the Massachusetts Institute of Technology, 1959.

[58] J.B. Roberts and P.D. Spanos, Random vibration and statistical linearization, Chichester, Wiley, 1990 (second edition, Dover Publications, Mineola, 2003)

[59] K. Magnus and K. Popp, Schwingungen, B.G. Teubner, 1997 (in German).

[60] I. Elishakoff and L. Andriamasy, Nonclassical Linearization Criteria in Nonlinear Stochastic Dynamics, Journal of Applied Mechanics, in press, 2009.

[61] L. Socha, Linearization Methods for Stochastic Dynamics Systems, Springer, Berlin, 2008.

[62] D.J. Ewins and D.J. Inman, Structural Dynamics at 2000: Currents Status and Future Directions, Research Studies Press, London, 2001.

[63] S. Braun, S.S. Rao and D.J. Ewins, Encyclopedia of vibration, Academic Press, 2002. 

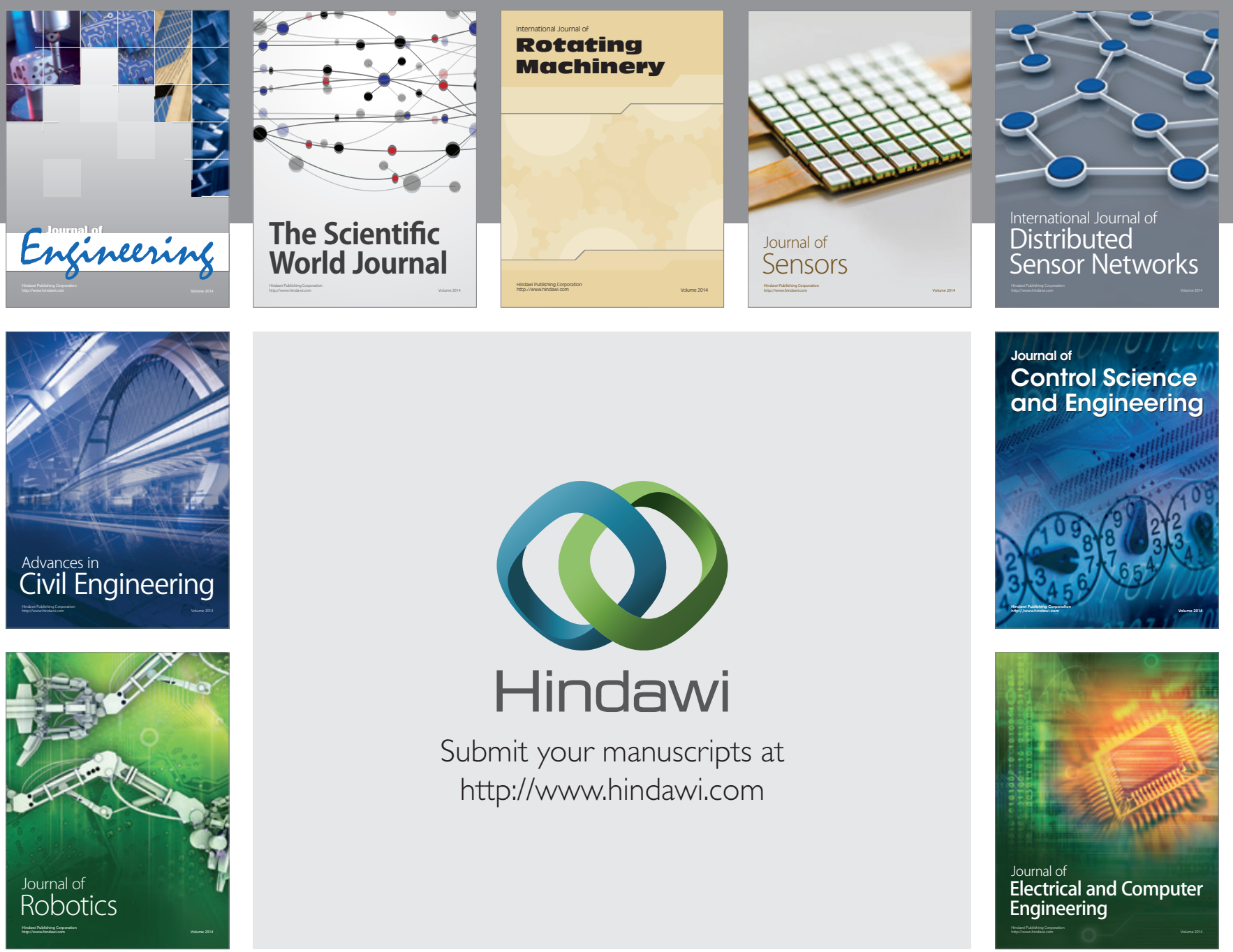

Submit your manuscripts at

http://www.hindawi.com
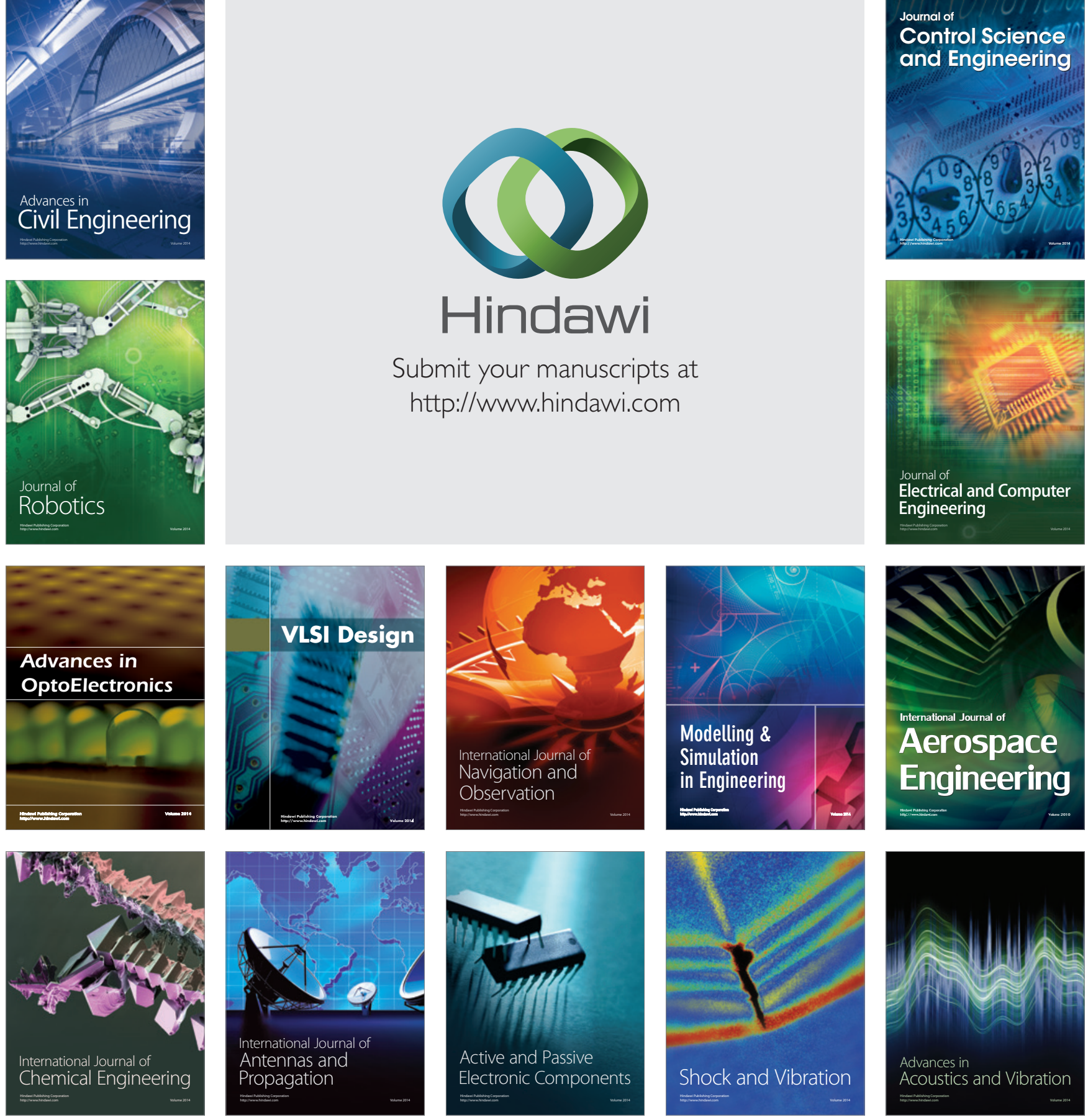